\title{
Comparison between esophageal and intestinal temperature responses to upper-limb exercise in individuals with spinal cord injury
}

\author{
Jason S. Au $\mathbb{1}^{1} \cdot$ Yoshi-ichiro Kamijo ${ }^{2}$ - Victoria L. Goosey-Tolfrey ${ }^{3} \cdot$ Christof A. Leicht $^{3} \cdot$ Maureen J. MacDonald ${ }^{1}$. \\ Yuki Mukai ${ }^{2} \cdot$ Fumihiro Tajima²
}

Received: 12 December 2018 / Revised: 31 January 2019 / Accepted: 1 February 2019 / Published online: 14 February 2019

( ) International Spinal Cord Society 2019

\begin{abstract}
Study design Experimental study.

Objective Individuals with spinal cord injuries (SCI) may present with impaired sympathetic control over thermoregulatory responses to environmental and exercise stressors, which can impact regional core temperature $\left(T_{\text {core }}\right)$ measurement. The purpose of this study was to investigate whether regional differences in $T_{\text {core }}$ responses exist during exercise in individuals with SCI.

Setting Rehabilitation centre in Wakayama, Japan.

Methods We recruited 12 men with motor-complete SCI (7 tetraplegia, 5 paraplegia) and 5 able-bodied controls to complete a 30-min bout of arm-cycling exercise at $50 \% \dot{\mathrm{VO}}_{2}$ peak reserve. $T_{\text {core }}$ was estimated using telemetric pills (intestinal temperature; $\left.T_{\text {int }}\right)$ and esophageal probes $\left(T_{\text {eso }}\right)$. Heat storage was calculated from baseline to 15 and 30 min of exercise. Results At $15 \mathrm{~min}$ of exercise, elevations in $T_{\text {eso }}\left(\Delta 0.39 \pm 0.22^{\circ} \mathrm{C} ; P<0.05\right)$, but not $T_{\text {int }}\left(\Delta 0.04 \pm 0.18{ }^{\circ} \mathrm{C} ; P=0.09\right)$, were observed in able-bodied men. At $30 \mathrm{~min}$ of exercise, men with paraplegia and able-bodied men both exhibited increases in $T_{\text {eso }}$ (paraplegia: $\Delta 0.56 \pm 0.30^{\circ} \mathrm{C}, P<0.05$; able-bodied men: $\Delta 0.60 \pm 0.31{ }^{\circ} \mathrm{C}, P<0.05$ ) and $T_{\text {int }}$ (paraplegia: $\Delta 0.38 \pm$ $0.33{ }^{\circ} \mathrm{C}, P<0.05$; able-bodied men: $\Delta 0.30 \pm 0.30^{\circ} \mathrm{C}, P<0.05$ ). $T_{\text {eso }}$ began rising $7.2 \mathrm{~min}$ earlier than $T_{\text {int }}$ (pooled, $P<0.01$ ). Heat storage estimated by $T_{\text {eso }}$ was greater than heat storage estimated by $T_{\text {int }}$ at $15 \min (P=0.02)$ and $30 \min (P=0.03)$ in men with paraplegia. No elevations in $T_{\text {eso }}, T_{\text {int }}$, or heat storage were observed in men with tetraplegia.

Conclusions While not interchangeable, both $T_{\text {eso }}$ and $T_{\text {int }}$ are sensitive to elevations in $T_{\text {core }}$ during arm-cycling exercise in men with paraplegia, although $T_{\text {eso }}$ may have superior sensitivity to capture temperature information earlier during exercise.
\end{abstract}

\section{Introduction}

Exercise-induced elevations in core body temperature $\left(T_{\text {core }}\right)$ are regulated within an acceptable range by the sweating and skin blood flow responses, mediated in part by the sympathetic nervous system in a negative feedback loop [1,

Jason S. Au

auj2@mcmaster.ca

1 Department of Kinesiology, McMaster University, Hamilton, Canada

2 Department of Rehabilitation, Wakayama Medical University, Wakayama, Japan

3 Peter Harrison Centre for Disability Sport, School of Sport, Exercise and Health Sciences, Loughborough University, Loughborough, UK
2]. While this is an adaptive process in able-bodied individuals, individuals with spinal cord injuries (SCI) may present with varying degrees of autonomic dysfunction as a result of damage to the spinal cord. Altered autonomic function has been shown to impair sympatheticallymediated regulatory processes below the lesion level, such as the thermoregulatory response to heat and exercise [3, 4].

Autonomic function in SCI may differentially affect the validity and sensitivity of different temperature measurement tools through alterations in regional thermoregulatory control. Rectal and esophageal temperatures $\left(T_{\text {eso }}\right)$ provide close estimates of $T_{\text {core }}$ during exercise, but are restricted to "lab-based" assessments that are not feasible to measure in the field $[5,6]$. Ingestible telemetric pills can provide a wireless measurement of intestinal temperature $\left(T_{\text {int }}\right)$, an estimate of $T_{\text {core }}$, and have been shown to be sensitive to heat- and exercise-related thermoregulatory challenges in both able-bodied men and individuals with SCI [7-9]. 
However, given the known differences in both gut motility [10] and abdominal skin temperature with higher level SCI $[11,12]$, it is unknown whether the telemetric pill provides equally sensitive assessment of $T_{\text {core }}$ in all individuals with SCI. Furthermore, much of the SCI thermoregulation research has been conducted in athletes with SCI [7, 11, 1316], with relatively little knowledge on the generalizability of methods to untrained adults with SCI who may have altered thermoregulatory sensitivity to exercise [17] and lower levels of heat production related to reduced aerobic capacity [18]. The ability to rely on non-invasive methods to estimate exercise- or environment-induced $T_{\text {core }}$ elevations is particularly important to untrained adults with SCI who are not habitually exposed to temperature stressors, and therefore may be at elevated risk for heat stress exposure.

The purpose of this study was to investigate whether $T_{\mathrm{int}}$ and $T_{\text {eso }}$ provide comparable information about the thermoregulatory response to upper-limb exercise in untrained men with paraplegia and tetraplegia. To simulate realistic exercise conditions in this population, $T_{\text {core }}$ sensitivity was tested during arm cycling at $50 \%$ of $\mathrm{VO}_{2}$ peak reserve, corresponding to moderate-to-vigorous intensity exercise recommended by the latest physical activity guidelines for adults with SCI [19]. Based on previous work in ablebodied individuals [9], we hypothesized that $T_{\text {int }}$ and $T_{\text {eso }}$ would both reflect exercise-related elevations in $T_{\text {core }}$ in individuals with paraplegia and tetraplegia.

\section{Methods}

\section{Experimental design}

Seventeen men were recruited for this study: seven individuals with tetraplegia, five individuals with paraplegia, and five able-bodied controls (Table 1 for participant characteristics). Participants were asked to come to the laboratory for two separate visits for a baseline testing session, as well as an acute exercise trial. During the first visit, degree of autonomic dysfunction was assessed by sympathetic skin responses (SSR) and the sit-up test to identify orthostatic hypotension $(\mathrm{OH})$, followed by a graded arm exercise test to exhaustion. At the second visit, participants performed 30 min of arm cycling exercise at $50 \% \mathrm{VO}_{2}$ peak, during which $T_{\text {core }}$ and skin temperature $\left(T_{\text {sk }}\right)$ were recorded throughout. The testing environment was maintained at $25^{\circ} \mathrm{C}$ at a relative humidity of $50 \%$.

\section{Sympathetic skin responses}

SSR testing was completed as previously described [20, 21], with modification. In the supine position, electrodes were placed on the dorsal and ventral sides of the left hand and foot, and were attached to a commercial data acquisition unit (PowerLab 26T (LTS), AD Instruments, Colorado Springs, CO, USA). In 30-40 s intervals, electrical stimulation was applied to the left median nerve, the left posterior tibial nerve, and the left supra-orbital nerve, in series. Five stimuli were applied at each nerve, with the pulse amplitude set to a threshold where a motor response could be elicited (12-20 mA, $0.2 \mathrm{~ms}$, single pulse). The presence or absence of a response was noted for each stimulation, which were summated at each site for SSR scoring.

\section{Sit-up test}

Participants were instrumented with an oscillometric blood pressure cuff (STBP-780, Colin, Komaki, Japan), and electrocardiogram for impedance estimation (PhysioFlow ${ }^{\circledR}$, NeuMeDx, Bristol, PA, USA). Participants were instructed to maintain a breathing rate of 15 breaths $/ \mathrm{min}$, timed to a metronome, to account for respiratory sinus arrhythmia. After 5 min of supine measurement, participants were passively sat up in a reclinable chair to a seated position for 15 min. Automated oscillometric blood pressure was recorded every minute, or until participant demonstrated signs of syncope. $\mathrm{OH}$ was defined as a drop in systolic blood pressure (SBP) of $\geq 20 \mathrm{mmHg}$, or a drop in diastolic blood pressure (DBP) of $\geq 10 \mathrm{mmHg}$ [22].

\section{$\dot{\mathrm{VO}}_{2}$ peak testing}

Peak oxygen consumption ( $\dot{\mathrm{VO}}_{2}$ peak) was assessed with a graded arm cycling test to volitional exhaustion. After $10 \mathrm{~min}$ of rest, participants were instructed to cycle at $50 \mathrm{rpm}$ on a manually-braked arm ergometer (Monark Rehab Trainer 881E; Monark Exercise AB, Varberg, Sweden) at 0, 5 , and $10 \mathrm{~W}$ for 3-min intervals, thereafter increasing the power output by $5 \mathrm{~W} / \mathrm{min}$ for individuals with tetraplegia or $10 \mathrm{~W} / \mathrm{min}$ for both individuals with paraplegia and ablebodied controls until test termination (cycling rate $<40 \mathrm{rpm}$ ). Ventilatory data were collected with a portable metabolic unit (MetaMax 3B, Cortex, Leipzig, Germany).

\section{Skin temperatures}

Skin temperatures were measured at 10 different sites with iButton sensors (DS1922T, Maxim Integrated Products, Inc., Sunnyvale, CA, USA): on the forehead and on the left side of the body at the chest, upper back, upper arm, forearm, dorsal hand, lower abdomen, anterior thigh, lateral calf, and dorsal foot. Data were sampled every $10 \mathrm{~s}$, which were then averaged every minute for analysis. $T_{\mathrm{sk}}$ was averaged between the arm, forearm, and hand for upperlimb temperature, and averaged between the thigh, leg, and foot for lower-limb temperature. In the absence of 
Table 1 Participant characteristics

\begin{tabular}{llll}
\hline Outcome & Tetraplegia $(n=7)$ & Paraplegia $(n=5)$ & Able-bodied $(n=5)$ \\
\hline Age (years) & $38 \pm 12$ & $53 \pm 7$ & $37 \pm 13$ \\
Height (m) & $1.72 \pm 0.07$ & $1.67 \pm 0.04$ & $1.69 \pm 0.05$ \\
Body mass (kg) & $57 \pm 14$ & $55 \pm 7$ & $64 \pm 14$ \\
Injury level range & $\mathrm{C} 4-\mathrm{C} 7$ & $\mathrm{~T} 4-\mathrm{T} 11$ & \\
AIS & & & \\
A & 4 & 5 & \\
B & 3 & 0 & \\
SSR & & & $5 / 5$ \\
$\quad$ Radial stimulation (hand/foot) & $1 / 0$ & $4 / 0$ & $5 / 5$ \\
$\quad$ Tibial stimulation (hand/foot) & $0 / 0$ & $0 / 0$ & $5 / 5$ \\
Supra-orbital stimulation (hand/foot) & $0 / 0$ & $3 / 0$ & $1 / 5$ \\
Cases of OH from SUT (/total) & $6 / 7$ & $0 / 5$ & \\
\hline
\end{tabular}

SSR are group averages, out of a maximum of five stimulations per limb. Values are mean \pm SD unless otherwise noted

AIS American Spinal Injury Association Impairment Scale, $O H$ orthostatic hypotension, RER respiratory exchange ratio, $R P E$ ratings of perceived exertion, $S S R$ sympathetic skin response, $S U T$ sit-up test, $V_{\mathrm{E}}$ ventilatory rate

SCI-specific $T_{\text {sk }}$ standards, mean $T_{\text {sk }}$ was calculated as per Ramanathan [23] by the following equation:

$$
\begin{aligned}
\text { Mean skin temperature }= & \left(0.3 \times T_{\text {chest }}\right)+\left(0.3 \times T_{\text {arm }}\right) \\
& +\left(0.2 \times T_{\text {thigh }}\right)+\left(0.2 \times T_{\text {calf }}\right)
\end{aligned}
$$

\section{Core temperature}

Core temperature was measured using both an ingestible telemetric capsule $\left(T_{\text {int }}\right)$, as well as an esophageal probe $\left(T_{\text {eso }}\right)$. The telemetric capsule was factory-calibrated and swallowed 8-10 h prior to arrival for the acute exercise trial (e-Celsius $^{\mathrm{TM}}$; BodyCap, Paris, France). Data were sampled every $30 \mathrm{~s}$ by a portable receiver (e-Performance; BodyCap), which were then averaged every minute for analysis. $T_{\text {eso }}$ was assessed by thermocouples in a polyethylene tube (PE90; Nippon Becton Dickinson Company, Tokyo, Japan), which was inserted through the right nostril and extended to the esophagus at the level of the right atria $\left(0.25^{*}\right.$ supine length), the placement of which was confirmed via a cold-water swallow. Immediately after each exercise trial, the esophageal probe was immersed in both room temperature and $40{ }^{\circ} \mathrm{C}$ water for calibration. The delay in temperature elevation was identified between the initiation of the test and the first sustained elevation in both $T_{\text {eso }}$ and $T_{\text {int. }}$.

\section{Heat storage}

Heat storage during exercise was calculated as per [24] by the following equation:
Heat storage $=\left(\left(0.8 \times\left[\Delta T_{\text {eso }}\right.\right.\right.$ or $\left.\left.\left.\Delta T_{\text {int }}\right)\right)+\left(0.2 \times \Delta T_{\text {sk }}\right)\right) \times c_{b}$

where $c_{b}$ is the specific heat capacity of body tissue $\left(3.49 \mathrm{~J} \mathrm{~g}^{-1}{ }^{\circ} \mathrm{C}^{-1}\right.$ ) and $T_{\mathrm{sk}}$ is the mean skin temperature as calculated from above. Heat storage was calculated from rest to each bout of exercise (ending at 15 and $30 \mathrm{~min}$ ) for both $T_{\text {eso }}$ and $T_{\text {int }}$.

\section{Exercise trial}

Exercise was performed in a temperature and humiditycontrolled room at $25^{\circ} \mathrm{C}$ and $50 \%$ humidity. Exercise intensity was chosen to reflect the most recent physical activity guidelines for individuals with SCI [19]. After instrumentation, participants were instructed to sit quietly for $10 \mathrm{~min}$ of rest, followed by two 15 -min bouts of exercise at $50 \% \mathrm{~V}_{2}$ reserve [25], separated by $2 \mathrm{~min}$ of rest. Throughout exercise, heart rate was monitored by singlelead ECG.

\section{Statistics}

Statistical analyses were performed using IBM SPSS Statistics (version 20.0.0; IBM Corp., Armonk, NY, USA). Data were visually assessed for normality using histograms and statistically using the Kolmogorov-Smirnov test. Resting characteristics and cardiopulmonary responses to exercise were compared between groups with one-way independent ANOVAs. One-way repeated measures ANOVAs were used to assess the change in $T_{\text {core }}$ and $T_{\text {sk }}$ at discrete intervals over the exercise bout within each group 
(i.e., timepoints: rest, $15 \mathrm{~min}$, recovery, $30 \mathrm{~min}$ ), where significant effects were followed by Tukey's HSD post-hoc test. Paired $t$-tests were used to examine the difference in heat storage between measurement types. Bland-Altman analysis was used to examine the difference between $T_{\text {int }}$ and $T_{\text {eso }}$ sensitivity during exercise, reporting the mean bias and $95 \%$ limits of agreement. Values are reported as mean \pm SD unless otherwise noted. In all analyses, the level of significance was set at $\alpha=0.05$.

\section{Results}

Participant characteristics and baseline autonomic function are described in Table 1. Autonomic testing revealed that men with tetraplegia had autonomic dysfunction as they presented with the absence of SSR and presence of $\mathrm{OH}$, while men with paraplegia had generally preserved upperlimb SSR responses and no $\mathrm{OH}$. As a methodological control, autonomic testing was also performed on ablebodied men, indicating intact SSR responses and only one case of diastolic $\mathrm{OH}$.

Peak and sub-maximal exercise responses are described in Table 2. There was a graded effect of lesion level on fitness, where men with SCI had a lower $\dot{\mathrm{VO}}_{2}$ peak than able-bodied men $(P<0.05)$. During the acute exercise bout, men with SCI worked at lower power output $(P<0.05)$ compared to ablebodied men, while men with tetraplegia had lower heart rate responses at both 15 and $30 \mathrm{~min}$ of exercise (both $P<0.01$ ), with no differences between men with paraplegia and ablebodied controls (both $P>0.90$ ).

At rest, there were no differences between tetraplegia, paraplegia, or able-bodied men in core temperature as measured by either $T_{\mathrm{int}}(P=0.14)$ or $T_{\mathrm{eso}}(P=0.35)$, although $T_{\text {eso }}$ was lower than $T_{\text {int }}$ by $0.63{ }^{\circ} \mathrm{C}$ for all individuals $\left(36.14 \pm 0.36{ }^{\circ} \mathrm{C}\right.$ vs. $\left.36.76 \pm 0.40{ }^{\circ} \mathrm{C}, P<0.01\right) . T_{\text {int }}$ and $T_{\text {eso }}$ demonstrated similar response patterns to exercise in men with paraplegia and able-bodied men, with both methods increasing by the end of the exercise bout $(P<$ 0.05 ; Fig. 1). $T_{\text {eso }}$ in able-bodied men demonstrated additional sensitivity to temperature changes at the 15-min and resting timepoints, without concurrent changes in $T_{\text {int }}$. There were no elevations in either $T_{\text {eso }}(P=0.24)$ or $T_{\text {int }}(P=$ 0.71 ), in men with tetraplegia. When all groups were pooled, we observed a delay in temperature elevations, where $T_{\text {eso }}$ began rising $7.2 \mathrm{~min}$ earlier than $T_{\text {int }}(5.2 \pm 1.9$ vs. $12.4 \pm 6.6 \mathrm{~min} ; P<0.01$ ). To assess the overall difference between temperature assessment sensitivity during exercise, a Bland-Altman analysis was conducted using the pooled data between the 15- and 30-min timepoints, separated by group (Fig. 2). An average bias of a $0.17^{\circ} \mathrm{C}$ greater $T_{\text {eso }}$ response was observed, with the $95 \%$ limits of agreement ranging from -0.25 to $0.59^{\circ} \mathrm{C}$.
Table 2 Cardiorespiratory outcomes during graded exercise test and exercise bout $($ mean $\pm \mathrm{SD})$

\begin{tabular}{|c|c|c|c|}
\hline Outcome & Tetraplegia & Paraplegia & Able-bodied \\
\hline \multicolumn{4}{|l|}{ Graded exercise test } \\
\hline Resting $\dot{\mathrm{VO}}_{2}\left(\mathrm{~L} \mathrm{~min}^{-1}\right)$ & $0.19 \pm 0.03^{\dagger}$ & $0.21 \pm 0.02$ & $0.25 \pm 0.04$ \\
\hline $\begin{array}{l}\text { Peak power output } \\
\text { (W) }\end{array}$ & $17 \pm 11 *^{\dagger}$ & $56 \pm 20$ & $77 \pm 11$ \\
\hline$\dot{\mathrm{V}}_{2}$ peak $\left(\mathrm{L} \mathrm{min}^{-1}\right)$ & $0.56 \pm 0.10^{* \dagger}$ & $1.11 \pm 0.31^{\dagger}$ & $1.64 \pm 0.28$ \\
\hline Peak heart rate (bpm) & $97 \pm 11 * \dagger$ & $161 \pm 19$ & $168 \pm 22$ \\
\hline Peak $V_{\mathrm{E}}\left(\mathrm{L} \min ^{-1}\right)$ & $26.8 \pm 5.2 * \dagger$ & $49.4 \pm 13.0^{\dagger}$ & $80.0 \pm 18.8$ \\
\hline Peak RER & $1.05 \pm 0.05^{\dagger}$ & $1.22 \pm 0.20$ & $1.39 \pm 0.22$ \\
\hline \multicolumn{4}{|c|}{ Exercise responses at $50 \% \dot{\mathrm{VO}}_{2}$ peak } \\
\hline Power output (W) & $7 \pm 2 * \dagger$ & $25 \pm 11^{\dagger}$ & $43 \pm 7$ \\
\hline Power output $\left(\% W_{\text {peak }}\right)$ & $47 \pm 17$ & $43 \pm 7$ & $55 \pm 3$ \\
\hline $\begin{array}{l}@ 15 \text { min Heart rate } \\
\text { (bpm) }\end{array}$ & $75 \pm 12 * \dagger$ & $131 \pm 14$ & $127 \pm 18$ \\
\hline @ 15 min RPE & $13 \pm 2$ & $15 \pm 1$ & $15 \pm 1$ \\
\hline $\begin{array}{l}\text { @ } 30 \text { min Heart rate } \\
\text { (bpm) }\end{array}$ & $77 \pm 6^{* \dagger}$ & $139 \pm 19$ & $138 \pm 21$ \\
\hline @30 min RPE & $14 \pm 2$ & $16 \pm 1$ & $16 \pm 1$ \\
\hline
\end{tabular}

$R P E$ ratings of perceived exertion, $V_{\mathrm{E}}$ ventilatory rate, $R E R$ respiratory exchange ratio

$* P<0.05$ vs. paraplegia

${ }^{\dagger} P<0.05$ vs. able-bodied

Average upper limb skin temperature did not significantly change from rest in any group $(P>0.05$; Fig. 3$)$, although forearm temperature exhibited a time $\times$ group interaction $(P<0.01)$, where temperature decreased in men with tetraplegia throughout the entire exercise bout $(\Delta$ $-1.80{ }^{\circ} \mathrm{C}$ from rest to $30 \mathrm{~min}$; all $P<0.05$ ). In contrast, lower limb skin temperature demonstrated a main effect for time $(P<0.01)$ where temperatures were lower during the exercise bout than during baseline rest for all participants $\left(\Delta-0.49^{\circ} \mathrm{C}\right.$ from rest to $30 \mathrm{~min}$; all $P<0.05$; Fig. 3 ).

Heat storage was increased when estimated from $T_{\text {eso }}$ compared to $T_{\text {int }}$ after the first $15 \mathrm{~min}$ of exercise in both men with paraplegia and able-bodied controls $(P<0.05$; Fig. 4). After the second bout of exercise, heat storage estimated by $T_{\text {eso }}$ was only greater in men with paraplegia $(P=0.03)$. Heat storage increased from the first bout to the second bout only in men with paraplegia ( $T_{\text {eso }}$ estimate $P=$ $0.03 ; T_{\text {int }}$ estimate $P<0.01$ ). There were no differences between heat storage method estimates or heat storage over time in men with tetraplegia $(P>0.05)$.

\section{Discussion}

We investigated whether two regional indicators of $T_{\text {core }}$ demonstrate similar responses during normothermic 

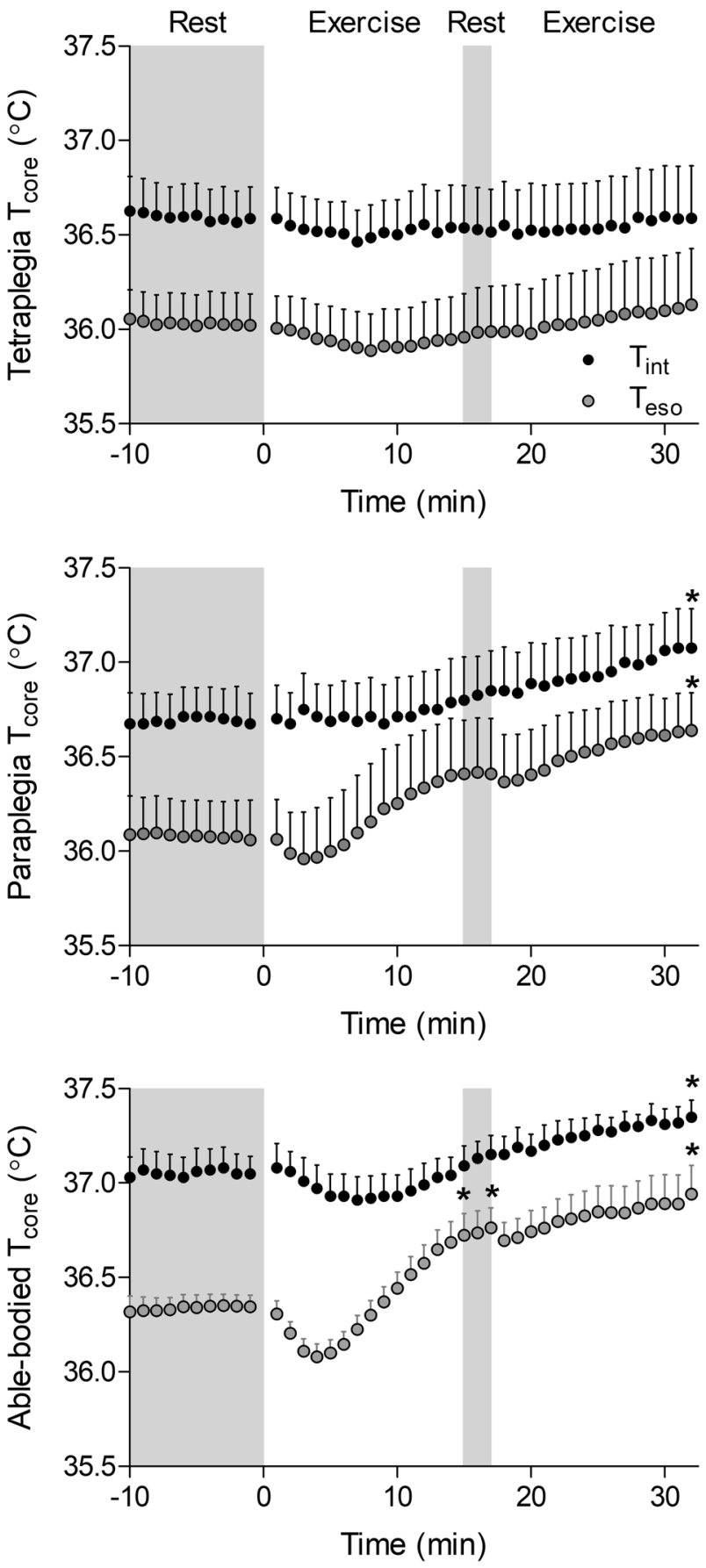

Fig. 1 Changes in core temperature $\left(T_{\text {core }}\right)$ during the exercise bout measured by esophageal (open circles; $T_{\text {eso }}$ ) and intestinal (closed circles; $\left.T_{\text {int }}\right)$ probes. Shaded areas indicate rest periods. Plotted points are mean + SEM. $* P<0.05$ different from resting temperature

exercise in individuals with different levels of SCI. The primary findings were: (1) amongst able-bodied men and men with paraplegia, both $T_{\text {eso }}$ and $T_{\text {int }}$ were sensitive to $T_{\text {core }}$ elevations during exercise in normothermia, with further $T_{\text {eso }}$ sensitivity in able-bodied men earlier during exercise; and (2) individuals with tetraplegia demonstrated

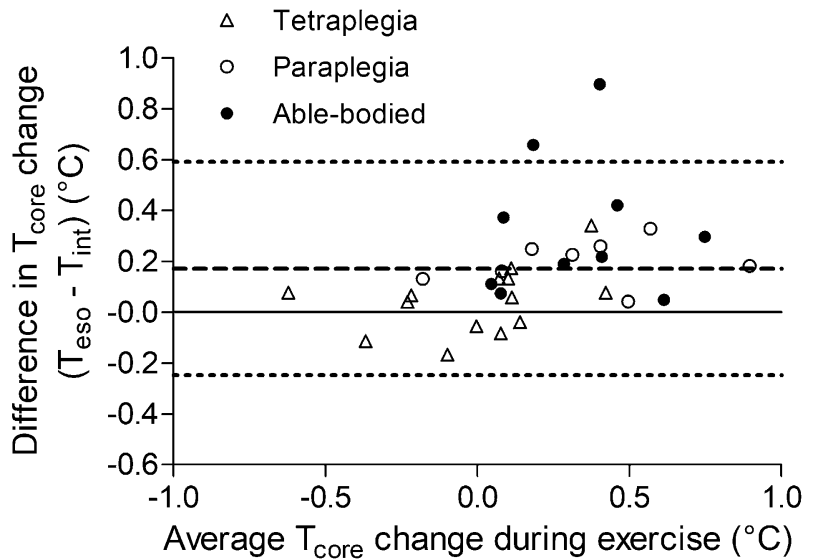

Fig. 2 Bland-Altman analysis indicating systematically larger changes in $T_{\text {eso }}$ compared to $T_{\text {int }}$ during exercise in men with tetraplegia, men with paraplegia, and able-bodied men. The difference between changes in temperature $\left(T_{\text {eso }}-T_{\text {int }}\right)$ is plotted against the range of average temperature changes, with the mean bias $\left(0.17^{\circ} \mathrm{C}\right)$ and $95 \%$ limits of agreement $\left(-0.25\right.$ to $\left.0.59^{\circ} \mathrm{C}\right)$ indicated by dashed lines
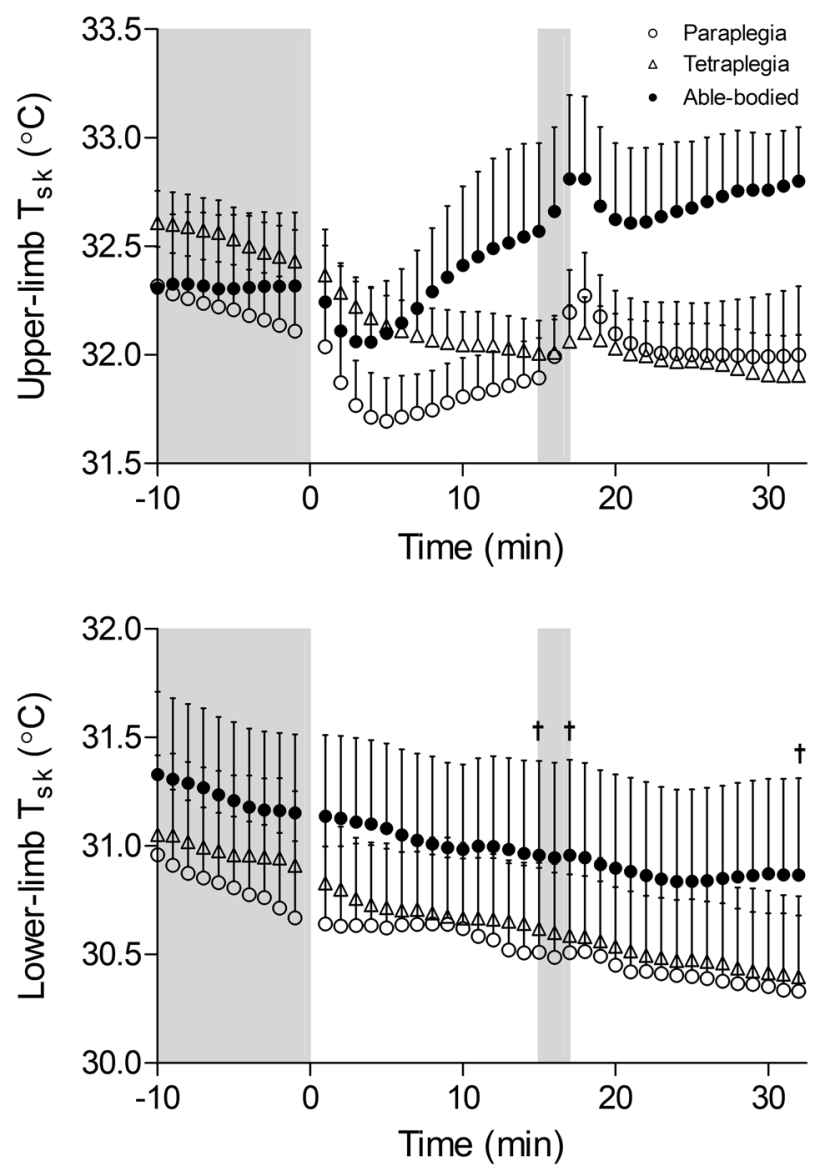

Fig. 3 Changes in upper (top) and lower (bottom) skin temperature $\left(T_{\mathrm{sk}}\right)$ during the exercise bout in individuals with tetraplegia (open triangles), paraplegia (open circles), and able-bodied men (closed circles). Shaded areas indicate rest periods. Plotted points are mean + SEM. ${ }^{\dagger} P<0.05$ all groups different from resting temperature 

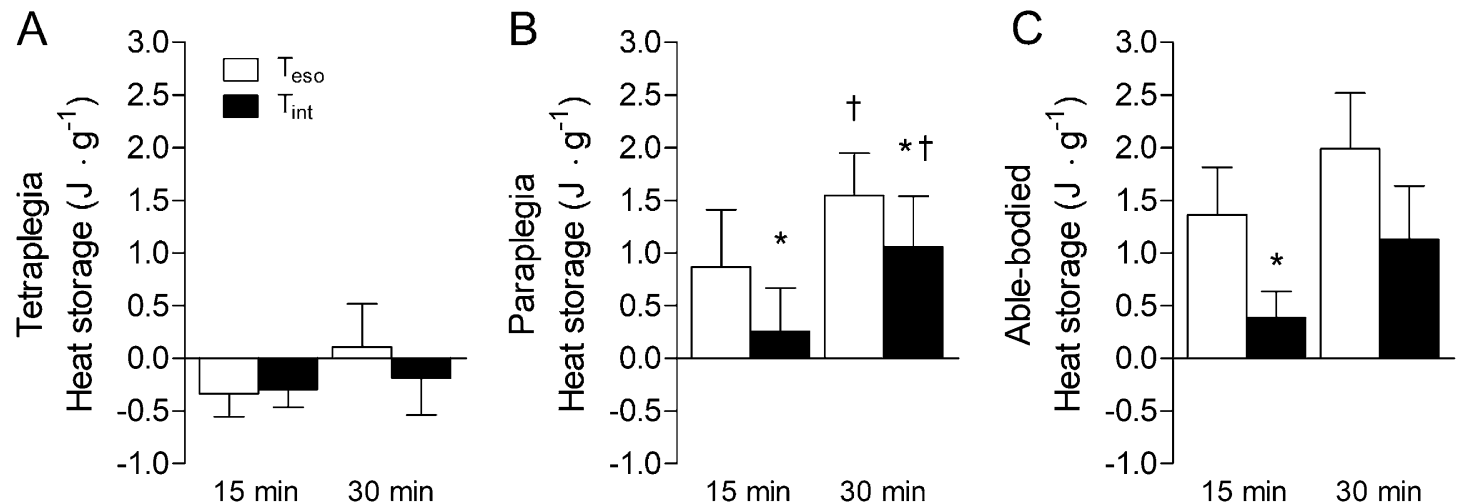

Fig. 4 Differences in heat storage estimated from esophageal $\left(T_{\text {eso }}\right)$ and intestinal $\left(T_{\text {int }}\right)$ temperatures after the first and second bouts of exercise in a men with tetraplegia, b men with paraplegia, and c

able-bodied men. Mean \pm SE. $* P<0.05$ different vs. $T_{\text {eso }} ;{ }^{\dagger} P<0.05$ different vs. $15 \mathrm{~min}$

signs of autonomic dysfunction at rest, and had no elevations in $T_{\text {eso }}$ or $T_{\text {int }}$ during 30 min of cycling at an intensity reflecting recommended workloads for healthy habitual physical activity. Our results indicate that $T_{\text {int }}$ and $T_{\text {eso }}$ can provide similar estimates of $T_{\text {core }}$ elevation during moderateintensity arm-cycling exercise in men with paraplegia, although our able-bodied data indicates $T_{\text {eso }}$ likely has superior sensitivity to capture temperature information earlier during exercise.

The use of telemetric pills provides a non-invasive estimate of $T_{\text {core }}$ which can be used for field assessments of thermoregulation during exercise in able-bodied individuals [9, 26, 27]. Ambulatory temperature monitoring is particularly important for individuals with SCI who may have temperature dysregulation secondary to autonomic dysfunction and are therefore at elevated risk for heat-related illness. Here, we report similar sensitivity in simultaneously measured $T_{\text {eso }}$ and $T_{\text {int }}$ during upper limb exercise in individuals with paraplegia and able-bodied men. $T_{\text {eso }}$ demonstrated an earlier rise during exercise, indicating rapid sensitivity to brief elevations in $T_{\text {core }}$. These findings agree with previous reports in able-bodied men that longer exercise durations are required to achieve a $0.1^{\circ} \mathrm{C}$ change in $T_{\text {int }}$ compared to $T_{\text {eso }}$ [28]. Circulating blood from the active limbs likely contributes to the robust temperature sensitivity, as warmed venous return from the working limbs directly impacts temperature conduction through the pulmonary artery to the level of the esophageal probe. Upperlimb exercise also results in greater heat production due to poor work efficiency (compared to the lower limbs), as well as less afferent input to the central nervous system resulting in greater heat storage [18]. It has been previously suggested that $T_{\text {int }}$ might be impacted by the location of the pill in the intestinal tract, and as such, there have been recommendations to standardize pill ingestion $6 \mathrm{~h}$ prior to measurement time for optimal intestinal transit time [9]. It is, however, unknown whether these assumptions hold true for individuals with SCI who have slower colon transit time (previously reported as SCI: $0.63 \pm 0.33 \mathrm{~cm} / \mathrm{h}$, controls: $2.58 \pm 1.20 \mathrm{~cm} / \mathrm{h}$ ) [29] and autonomically-mediated peristaltic dysfunction [30]. Previous studies in able-bodied men and athletes with SCI have demonstrated minimal differences between $T_{\text {core }}$ estimations using esophageal, intestinal, or rectal probes during high-intensity exercise [27, 31]. Our findings are likely specific to individuals with SCI with poor aerobic fitness performing moderate-intensity exercise, which represents the fitness and activity of the majority of untrained adults with SCI [32]. It is unknown whether exercise in heat or longer duration exercise would diminish observed differences between methods in men with paraplegia, although an exercise duration of $30 \mathrm{~min}$ is a realistic and relevant physiological challenge for most individuals with SCI.

While the thermoregulatory responses to prolonged exercise have previously been documented in athletes with SCI [7, 8, 11, 13-16, 31, 33, 34], relatively little is known about the exercise response in untrained adults with SCI, in particular, individuals with tetraplegia. Using a moderateintensity exercise protocol recommended by national SCI physical activity guidelines [19], we observed increases in $T_{\text {core }}$ in men with paraplegia and able-bodied controls, but not in individuals with tetraplegia, likely due to lower exercise power output amongst men with tetraplegia. The exercise intensity levels in individuals with tetraplegia were likely below a threshold required to generate sufficient heat stress as evidenced by a lack of elevations in upper-limb $T_{\text {sk }}$ or heat storage during exercise in a normothermic environment. Low cardiorespiratory loads at a similar relative exercise intensity were evident by both the lower exercise heart rate as well as moderate RPE scores during the final minute of exercise, likely as a result of autonomic dysfunction (i.e., lack of SSR, symptoms of $\mathrm{OH}$ ) and low cardiorespiratory fitness. Previously, Webborn et al. [33] demonstrated robust increases in $T_{\text {int }}$ in athletes with 
tetraplegia in response to an intermittent sprint exercise protocol on an arm ergometer, albeit at four times the power output of our untrained participants. Although our data do not indicate heat stress in men with tetraplegia and poor cardiorespiratory fitness, it remains unknown whether environmental heat stress would impact the effect of exercise on regional elevations in $T_{\text {core }}$ in this population.

Telemetric measurement of $T_{\text {core }}$ in individuals with SCI is important for the dynamic assessment of thermoregulation, a key homeostatic process that may be impaired by autonomic dysfunction following SCI. In our study, we observed similar increases in $T_{\text {eso }}$ and $T_{\text {int }}$ during $30 \mathrm{~min}$ of upper-limb exercise in men with paraplegia as well as ablebodied controls. The absence of exercise-induced heat stress in men with tetraplegia, as reflected by a lack of increases in $T_{\text {core }}$ or $T_{\text {sk }}$, was likely due to limitations to aerobic capacity and low power outputs during the acute exercise. Our results indicate that $T_{\text {int }}$ and $T_{\text {eso }}$ can provide similar estimates of $T_{\text {core }}$ elevation during moderate-intensity armcycling exercise in men with paraplegia.

\section{Data availability}

The datasets generated during the current study are available from the corresponding author on reasonable request.

Acknowledgements The authors gratefully acknowledge the contributions to this study by Kyungsu Jung as well as the medical doctors and physiotherapists of Wakayama Medical University.

Funding This study was supported by funding from the Japan Society for the Promotion of Science through a Mitacs partnership, as well as the Natural Sciences and Engineering Research Council of Canada (DG No. 238819-13 to MJM). Equipment was provided through the Peter Harrison Centre for Disability Sport (Loughborough University).

Author contributions JSA was responsible for designing the protocol, conducting the experiments, extracting and analyzing the data, interpreting the results, as well as drafting the final manuscript. YK was responsible for designing the protocol, conducting the experiments, extracting the data, interpreting the results, as well as providing feedback on the final manuscript. VLG-T was responsible for designing the protocol, interpreting the study findings, as well as providing feedback on the final manuscript. CAL was responsible for designing the protocol, interpreting the study findings, as well as providing feedback on the final manuscript. MJM was responsible for designing the protocol, interpreting the study findings, as well as drafting the final manuscript. YM was responsible for conducting the experiments, extracting the data, as well as providing feedback on the final manuscript. FT was responsible for designing the protocol, interpreting the study findings, as well as providing feedback on the final manuscript.

\section{Compliance with ethical standards}

Conflict of interest The authors declare that they have no conflict of interest.
Ethics approval All methods and procedures were approved by the Medical Ethical Committee of Wakayama Medical University (\#2076), adhering to the Declaration of Helsinki except for registration in a database. All testing was completed in the Wakayama Medical University Genki Development Institute (Wakayama, Japan). All participants gave verbal and written consent prior to participation in this study. We certify that all applicable institutional and governmental regulations concerning the ethical use of human volunteers were followed during the course of the research.

Publisher's note: Springer Nature remains neutral with regard to jurisdictional claims in published maps and institutional affiliations.

\section{References}

1. Greenleaf JE, Castle BL. Exercise temperature regulation in man during hypohydration and hyperhydration. J Appl Physiol. 1971;30:847-53.

2. Low DA, Keller DM, Wingo JE, Brothers RM, Crandall CG. Sympathetic nerve activity and whole body heat stress in humans. J Appl Physiol. 2011;111:1329-34.

3. Krassioukov A. Autonomic function following cervical spinal cord injury. Respir Physiol Neurobiol. 2009;169:157-64.

4. Krassioukov AV, Karlsson A-K, Wecht JM, Wuermser L-A, Mathias CJ, Marino RJ. Assessment of autonomic dysfunction following spinal cord injury: rationale for additions to International Standards for Neurological Assessment. J Rehabil Res Dev. 2007;44:103-12.

5. Shiraki K, Konda N, Sagawa S. Esophageal and tympanic temperature responses to core blood temperature changes during hyperthermia. J Appl Physiol. 1986;61:98-102.

6. Saltin B, Hermansen L. Esophageal, rectal, and muscle temperature during exercise. J Appl Physiol. 1966;21:1757-62.

7. Griggs KE, Leicht CA, Price MJ, Goosey-Tolfrey VL. Thermoregulation during intermittent exercise in athletes with a spinal cord injury. Int J Sports Physiol Perform. 2014;10:469-75.

8. Griggs KE, Havenith G, Price MJ, Mason BS, Goosey-Tolfrey VL. Thermoregulatory responses during competitive wheelchair rugby match play. Int J Sports Med. 2017;38:177-83.

9. Byrne C, Lim CL. The ingestible telemetric body core temperature sensor: a review of validity and exercise applications. Br J Sports Med. 2007;41:126-33.

10. Lynch A, Antony A, Dobbs B, Frizelle F. Bowel dysfunction following spinal cord injury. Spinal Cord. 2001;39:193-203.

11. Price MJ, Campbell IG. Effects of spinal cord lesion level upon thermoregulation during exercise in the heat. Med Sci Sport Exerc. 2003;35:1100-7.

12. Song YG, Won YH, Park SH, Ko MH, Seo JH. Changes in body temperature in incomplete spinal cord injury by digital infrared thermographic imaging. Ann Rehabil Med. 2015;39:696-704.

13. Price MJ, Campbell IG. Thermoregulatory responses of paraplegic and able-bodied athletes at rest and during prolonged upper body exercise and passive recovery. Eur J Appl Physiol Occup Physiol. 1997;76:552-60.

14. Price MJ, Campbell IG. Thermoregulatory responses of spinal cord injured and able-bodied athletes to prolonged upper body exercise and recovery. Spinal Cord. 1999;37:772-9.

15. Goosey-Tolfrey V, Swainson M, Boyd C, Atkinson G, Tolfrey K. The effectiveness of hand cooling at reducing exercise-induced hyperthermia and improving distance-race performance in wheelchair and able-bodied athletes. J Appl Physiol. 2008; $105: 37-43$. 
16. Veltmeijer MT, Pluim B, Thijssen DH, Hopman MT, Eijsvogels TM. Thermoregulatory responses in wheelchair tennis players: a pilot study. Spinal Cord. 2014;52:373-7.

17. Lorenzo S, Minson CT. Heat acclimation improves cutaneous vascular function and sweating in trained cyclists. J Appl Physiol. 2010;109:1736-43.

18. Minson CT, Brunt VE. Thermoregulatory considerations for the performance of exercise in SCI. In: Taylor J, editor. The physiology of exercise in spinal cord injury. Boston, MA, USA: Springer; 2016. p. 127-60.

19. Martin Ginis KA, van der Scheer JW, Latimer-Cheung AE, Barrow A, Bourne C, Carruthers $\mathrm{P}$, et al. Evidence-based scientific exercise guidelines for adults with spinal cord injury: an update and a new guideline. Spinal Cord. 2018;56:308-21.

20. Claydon VE, Krassioukov AV. Orthostatic hypotension and autonomic pathways after spinal cord injury. J Neurotrauma. 2006;23:1713-25.

21. Hubli M, Krassioukov AV. How reliable are sympathetic skin responses in subjects with spinal cord injury? Clin Auton Res. 2015;25:117-24.

22. The Consensus Committee of the American Autonomic Society and the American Academy of Neurology. Consensus statement on the definition of orthostatic hypotension, pure autonomic failure and multiple system atrophy. Neurology. 1996;46:1470.

23. Ramanathan NL. A new weighting system for mean surface temperature of the human body. J Appl Physiol. 1964;19:531-3.

24. Havenith G, Inoue Y, Luttikholt V, Kenney WL. Age predicts cardiovascular, but not thermoregulatery, responses to humid heat stress. Eur J Appl Physiol Occup Physiol. 1995;70:88-96.

25. Swain DP, Franklin BA. $\mathrm{VO}_{2}$ reserve and the minimal intensity for improving cardiorespiratory fitness. Med Sci Sport Exerc. 2002;34:152-7.
26. Lee SMC, Williams WJ, Schneider SM. Core temperature measurement during submaximal exercise: esophageal, rectal, and intestinal temperatures. NASA Center for AeroSpace Information Technical Report, NASA/TP 210133; 2000. https://ntrs.nasa.gov/ search.jsp?R=20000036595.

27. Gant N, Atkinson G, Williams C. The validity and reliability of intestinal temperature during intermittent running. Med Sci Sports Exerc. 2006;38:1926-31.

28. Kolka MA, Quigley MD, Blanchard LA, Toyota DA, Stephenson LA. Validation of a temperature telemetry system during moderate and strenuous exercise. J Therm Biol. 1993;18:203-10.

29. Keshavarzian A, Barnes WE, Bruninga K, Nemchausky B, Mermall H, Bushnell D. Delayed colonic transit in spinal cord-injured patients measured by indium-111 amberlite scintigraphy. Am J Gastroenterol. 1995;90:1295-300.

30. Krassioukov A, Eng JJ, Claxton G, Sakakibara BM, Shum S. Neurogenic bowel management after spinal cord injury: a systematic review of the evidence. Spinal Cord. 2010;48:718-33.

31. Pritchett RC, Green JM, Pritchett KL, Bishop P. Heat storage in upper and lower body during high-intensity exercise in athletes with spinal cord injuries. South Afr J Sport Med. 2011;23: 9-13.

32. Simmons OL, Kressler J, Nash MS. Reference fitness values in the untrained spinal cord injury population. Arch Phys Med Rehabil. 2014;95:2272-8.

33. Webborn N, Price MJ, Castle PC, Goosey-Tolfrey VL. Effects of two cooling strategies on thermoregulatory responses of tetraplegic athletes during repeated intermittent exercise in the heat. $\mathrm{J}$ Appl Physiol. 2005;98:2101-7.

34. Griggs K, Havenith G, Price M, Paulson T, Goosey-Tolfrey V. Effectiveness of pre-cooling and cooling during play on wheelchair rugby performance. Extrem Physiol Med. 2015;4:A4. 